\title{
Does Early Cerebral Near-Infrared Spectroscopy Monitoring Predict Outcome in Neonates with Hypoxic Ischaemic Encephalopathy? A Systematic Review of Diagnostic Test Accuracy
}

\author{
Aisling A. Garvey ${ }^{a, b}$ Andreea M. Pavel ${ }^{a, b}$ Deirdre M. Murray ${ }^{a, b}$ \\ Geraldine B. Boylan ${ }^{a, b}$ Eugene M. Dempsey ${ }^{a, b}$ \\ aINFANT Research Centre, Cork, Ireland; ' Department of Paediatrics and Child Health, University College Cork, Cork, \\ Ireland
}

\section{Keywords}

Near-infrared spectroscopy · Hypoxic ischaemic

encephalopathy · Early biomarker · Neonatal

encephalopathy

\begin{abstract}
Introduction: Hypoxic ischaemic encephalopathy (HIE) remains one of the top 10 contributors to the global burden of disease. Early objective biomarkers are required. Near-infrared spectroscopy (NIRS) may provide a valuable insight into cerebral perfusion and metabolism. We aimed to determine whether early NIRS monitoring ( $<6 \mathrm{~h}$ of age) can predict outcome as defined by grade of encephalopathy, brain MRI findings, and/or neurodevelopmental outcome at 1-2 years in infants with HIE. Methods: We searched PubMed, Scopus, Web of Science, Embase, and The Cochrane Library databases (July 2019). Studies of infants born $\geq 36^{+0}$ weeks gestation with HIE who had NIRS recording commenced before $6 \mathrm{~h}$ of life were included. We planned to provide a narrative of all the studies included, and if similar clinically and methodologically, the results would be pooled in a meta-analysis to determine test accuracy. Results: Seven studies were included with a combined total of 161 infants. Only 1 study includ-
\end{abstract} probes were utilized with varying outcome measures making comparison difficult. Although some studies showed a trend towards higher $\mathrm{CSO}_{2}$ values before $6 \mathrm{~h}$ in infants with adverse neurodevelopmental outcomes, in the majority, this was not significant until beyond $24 \mathrm{~h}$ of life. Conclusion: Very little data currently exists to assess the use of early NIRS to predict outcome in infants with HIE. Further studies using a standardized approach are required before NIRS can be evaluated as a potential objective assessment tool for early identification of at-risk infants.

(c) 2021 S. Karger AG, Base

\section{Introduction}

Hypoxic ischaemic encephalopathy (HIE) is the leading cause of acquired brain injury in term newborns and accounts for 1-3/1,000 live births [1]. HIE results from an initial insult due to cerebral hypoxia and ischaemia resulting in energy depletion and lactate accumulation secondary to anaerobic metabolism. This is followed by a secondary insult, termed the reperfusion injury, following circulatory restoration $[2,3]$. HIE is clinically graded 
as mild, moderate, and severe based on neurological examination after birth, and long-term neurodevelopmental outcome correlates with grade of encephalopathy. Therapeutic hypothermia (TH) is the current approved treatment for infants with moderate and severe grades of HIE and should be commenced within $6 \mathrm{~h}$ of life, before the secondary energy failure commences, to be most effective [3-5]. Historically, infants with mild grades of encephalopathy were thought to have normal outcomes [6]; however, it is becoming more apparent that these infants are at increased risk of adverse neurodevelopmental outcome, specifically significant levels of learning, emotional, and behavioural difficulties [7].

This presents significant challenges as the current methods of identifying such infants at risk of long-term neurodevelopmental difficulties are somewhat limited. It is clear that some infants who have difficulties later are currently falling below the threshold for therapeutic intervention [7]. The earliest available tools including Apgar score, $\mathrm{pH}$, and lactate levels do not correlate with outcome, rather they succeed in focusing attention on newborns at risk of HIE [8-10].

Currently, assessment is centred around clinical evaluation, and the modified Sarnat and Thompson scores [6, 11] are most commonly used in clinical practice. Originally validated to examine infants repeatedly in the first week of life $[6,11]$, when used in the first $6 \mathrm{~h}$ of life, they can be influenced by factors including mode of delivery and maternal analgesia.

Electroencephalography (EEG) plays a key role in both prognostication and detection of seizures in infants with HIE. Amplitude-integrated EEG (aEEG) in the first $6 \mathrm{~h}$ of life was the most useful tool in predicting outcome in the pre-TH era; however, this predictive ability has now altered if $\mathrm{TH}$ is commenced very early, and the best predictive ability is now seen at $48 \mathrm{~h}$ [12]. Continuous, multichannel EEG requires expert interpretation, and most units do not have 24-h access to a neonatal neurophysiologist or neurologist.

Hypoxic injury is not evident on conventional MRI until the first to second week of life [13] and is therefore not helpful in detecting or grading encephalopathy in the early postnatal period [14]. Early MRI scans may be helpful in ascertaining the timing of the initial injury [15-17], although there were concerns that early diffusion weighted imaging scans may underestimate the extent of the injury $[17,18]$.

Prediction of long-term outcome remains challenging, and it is important to consider other confounding factors in the neonatal unit and beyond. However, at present, we have no single test to accurately identify infants at risk of long-term neurodevelopmental outcome in the setting of HIE. Alternative methods of identification are thus required.

$\mathrm{HI}$ injury results in a period of hypoperfusion at the time of initial insult followed by circulatory restoration [3]. Cerebral blood flow (CBF) has been shown to increase in the first $24 \mathrm{~h}$ following $\mathrm{HI}$ injury likely due to a disruption in haemodynamic control $[19,20]$. This early hyperperfusion has been shown to be associated with increased risk of brain injury [21]. Clinicians commonly associate time of injury with time of birth, which is not always the case and may explain why studies on the use of early near-infrared spectroscopy (NIRS) as a prognostic tool are conflicting, but beyond $24 \mathrm{~h}$ of life, NIRS, specifically cerebral oxygenation $\left(\mathrm{cSO}_{2}\right)$ and metabolism, appears to be a good predictor of neurodevelopmental outcome $[22,23]$. Data on NIRS in the first $6 \mathrm{~h}$ of life (within the therapeutic window) may aid in prognostication and objective identification of cases for therapeutic intervention. The aim of this systematic review is to determine whether early cerebral NIRS monitoring ( $<6 \mathrm{~h}$ of age) can predict neonatal outcome as defined by grade of encephalopathy (defined clinically using Thompson or Sarnat scoring or electrophysiologically using visual EEG analysis), brain MRI findings, and/or neurodevelopmental outcome at 1-2 years of age.

\section{Methods}

As per the Preferred Items for Systematic Reviews and MetaAnalysis Statement, a systematic stepwise approach was taken to search various databases [24].

\section{Search Strategy}

Relevant articles were identified from the following databases by 2 authors, A.G. and A.P.: PubMed, Scopus, Web of Science, Embase, and The Cochrane Library. Searches were filtered to include human studies published in the English language in the last 30 years (January 1988 to July 2019). Three broad search terms were used (Neonate, HIE, and NIRS) along with their corresponding synonyms and MeSH terms (online suppl. Table. 1; for all online suppl.material, seewww.karger.com/doi/10.1159/000518687). Additional published reports identified in review articles or screened articles were subsequently included.

\section{Study Selection}

All articles were indexed to EndNote, and duplicates were removed. Articles were initially screened based on the title and then by abstract by 1 author (A.G.). The remaining articles were then screened independently for inclusion criteria by both A.G. and E.D., and any discrepancies were discussed and resolved. 


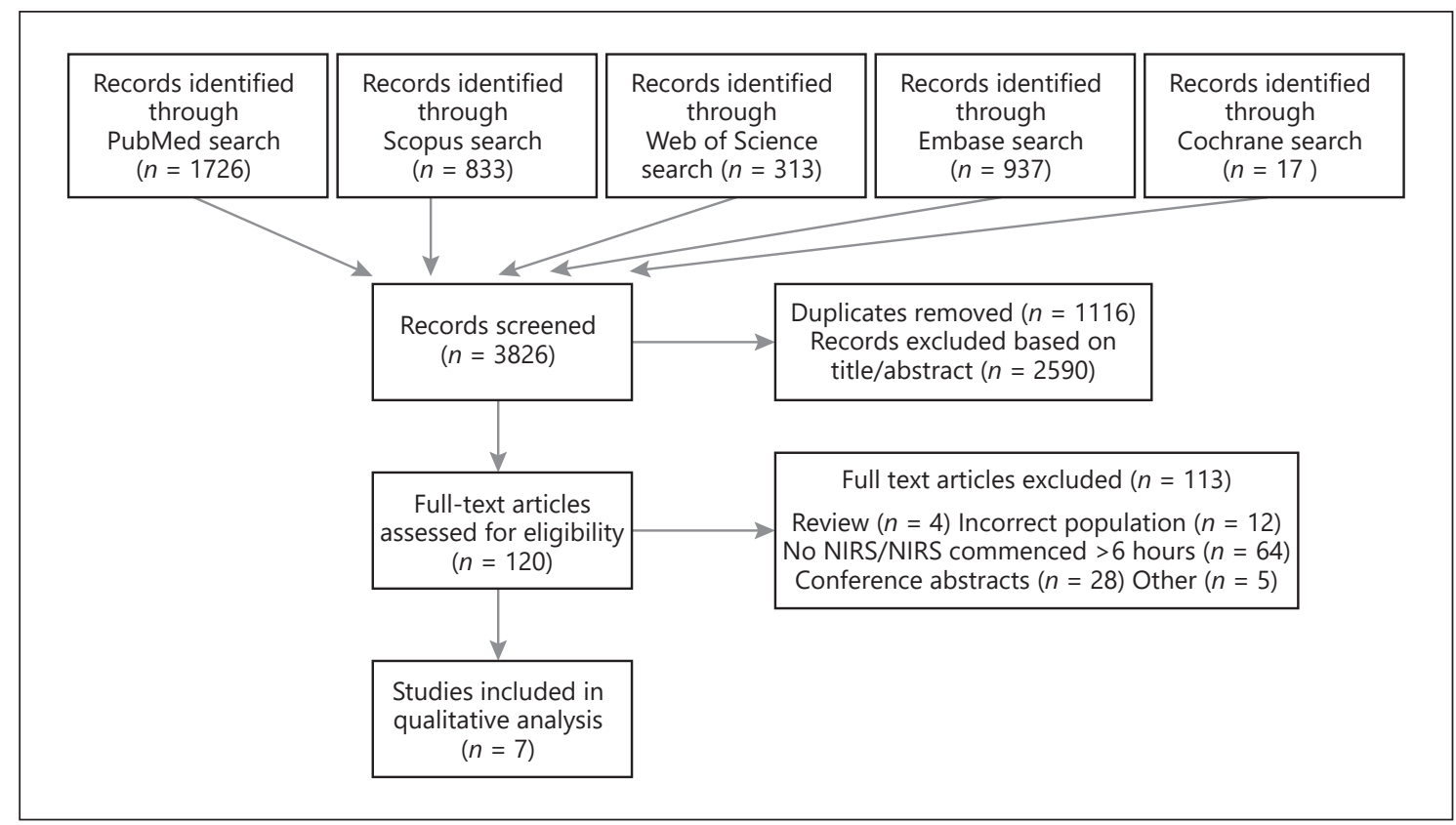

Fig. 1. PRISMA diagram of literature search. The PRISMA diagram displays the number of articles identified from each database searched, screening process, and number of studies and reasons for subsequent exclusion from analysis. NIRS, near-infrared spectroscopy.

Inclusion criteria consisted of the following:

1. Observational cohort study or clinical randomized or quasirandomized controlled trials.

2. Human study.

3. Newborn infants born at $\geq 36$ weeks gestational age.

4. Neonatal HIE defined clinically, using Thompson or Sarnat scoring, and/or electrophysiologically, using amplitude-integrated (aEEG) or continuous EEG (cEEG).

5. Cerebral NIRS monitoring commenced early, defined as before $6 \mathrm{~h}$ of age.

Animal studies, studies that were not in English, or conference abstracts were not included.

\section{Strategy for Data Analysis}

We planned to include a narrative of all the studies included, detailing the early findings of NIRS monitoring. If the studies were similar clinically and methodologically, we planned to pool the results of the studies in a meta-analysis. Data from each study would be summarized in a $2 \times 2$ table (true-positives, false-positives, false-negatives, and true negatives) and the sensitivity and specificity for each study calculated. A coupled forest plot displaying the sensitivity and specificity and corresponding 95\% CIs for each study and a summary plot displaying the sensitivity-specificity point for each study would be created. Heterogeneity among studies would be investigated by visual inspection of these plots. If no substantial heterogeneity was observed, pooled sensitivity and specificity estimates and corresponding $95 \%$ CIs would be calculated for studies with a common threshold using the bivariate random effects regression model or the univariate random-effects model (if the number of studies is small). If different thresholds were reported in the individual studies, a hierarchical summary receiver operating characteristic curve would be created and a global summary of test accuracy calculated. The analysis would be performed using Stata (version 13.0; StataCorp LP, College Station, TX, USA).

\section{Risk of Bias (Quality) Assessment}

Studies were critically appraised using the Quality Assessment of Diagnostic Accuracy Studies (QUADAS-2) tool to assess their validity and applicability. Each article was scored by both reviewers independently. The review protocol was published on the International prospective register of systematic reviews (PROSPE$\mathrm{RO}$ ) prior to conducting database search (Registration No. PROSPERO 2019 CRD42019127907).

\section{Results}

Our initial search identified 3,826 articles. Figure 1 provides a flow diagram of the search results.

From these articles, 1,116 duplicates were removed and 2,590 articles were excluded based on title or abstract. One hundred and twenty full-text articles were assessed for eligibility criteria, and from these, we identified 7 original articles that described the use of NIRS in infants with HIE within the first $6 \mathrm{~h}$ of life. The details of included articles are summarized in Table 1 . The principle reasons 


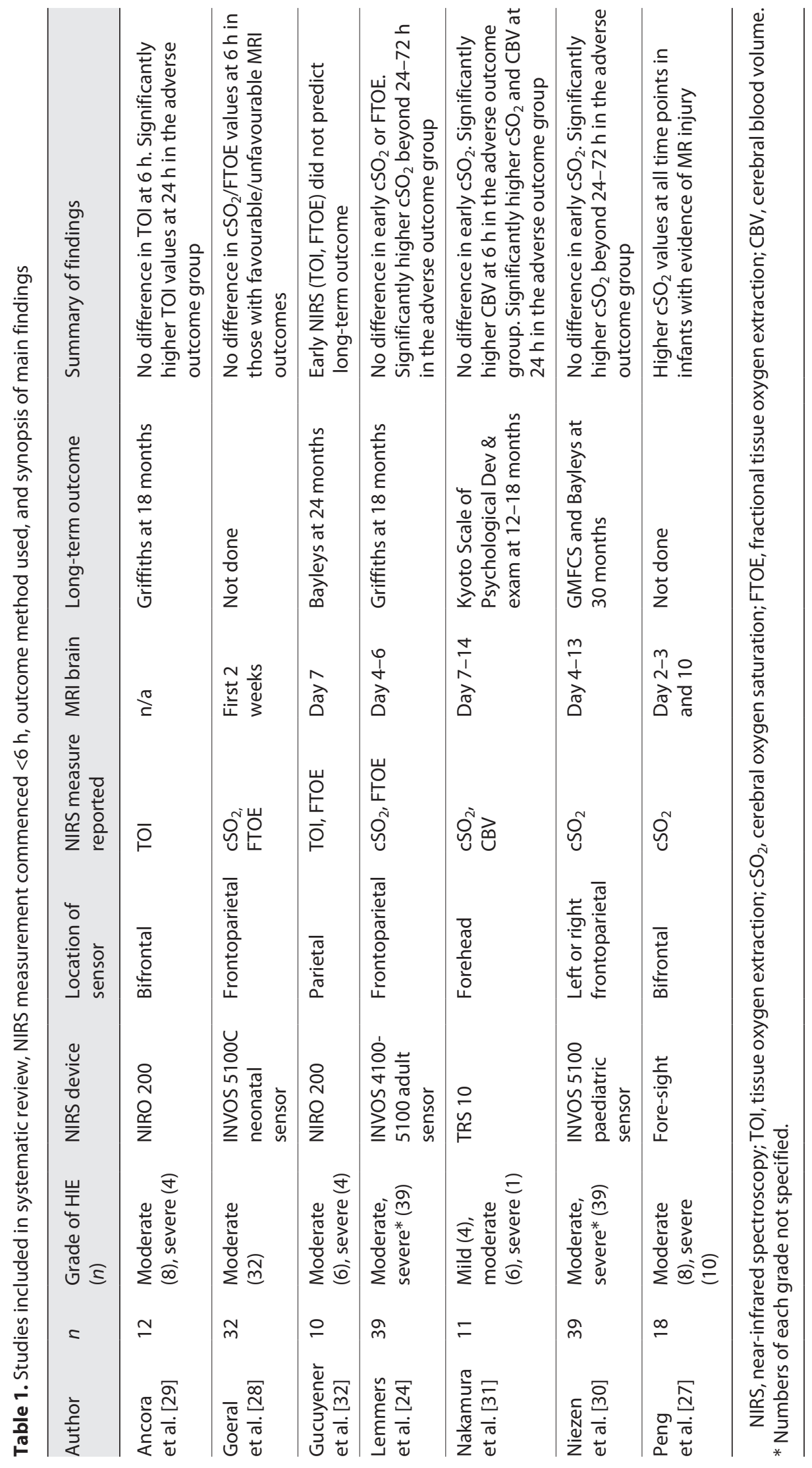

4 
Table 2. Assessment of risk of bias of included studies using quality assessment of diagnostic accuracy tests -2

\begin{tabular}{|c|c|c|c|c|c|c|c|}
\hline & \multicolumn{4}{|c|}{ Risk of bias } & \multicolumn{3}{|c|}{ Applicability concerns } \\
\hline & $\begin{array}{l}\text { patient } \\
\text { selection }\end{array}$ & $\begin{array}{l}\text { index } \\
\text { test }\end{array}$ & $\begin{array}{l}\text { reference } \\
\text { standard }\end{array}$ & $\begin{array}{l}\text { flow and } \\
\text { timing }\end{array}$ & $\begin{array}{l}\text { patient } \\
\text { selection }\end{array}$ & $\begin{array}{l}\text { index } \\
\text { test }\end{array}$ & $\begin{array}{l}\text { reference } \\
\text { standard }\end{array}$ \\
\hline Ancora et al. [29] & Low & Unclear & Low & Low & Low & Low & Low \\
\hline Goeral et al. [28] & Low & Unclear & Low & Low & Low & Low & Low \\
\hline Gucuyener et al. [32] & Unclear & Unclear & Unclear & High & Low & Low & Low \\
\hline Lemmers et al. [24] & Low & Unclear & Low & Low & Low & Low & Low \\
\hline Nakamura et al. [31] & High & Unclear & High & Low & Low & Low & Low \\
\hline Niezen et al. [30] & Low & Unclear & Unclear & Low & Low & Low & Low \\
\hline Peng et al. [27] & Unclear & Unclear & Low & Low & Unclear & Low & Low \\
\hline
\end{tabular}

for non-inclusion are outlined in online supplementary Table 2, but predominantly were related to a NIRS device not being used (e.g., MRI to measure cerebral metabolism and blood flow), start time of NIRS recording not documented, or NIRS monitoring commenced after $6 \mathrm{~h}$ of life.

\section{Included Studies}

Details of included studies are outlined in Table 1, and a brief narrative is included below.

\section{Early NIRS to Determine Grade of Encephalopathy}

In all of the included studies, grade of encephalopathy was determined at or before time of enrolment using standardized assessment tools such as the Sarnat or Thompson score. Six of the 7 trials focused on moderate to severe encephalopathy only. Early NIRS was not assessed for its ability to predict grade of encephalopathy nor were they correlated with the individual grades. Only 1 study included infants with mild encephalopathy, and no study included a control group.

\section{Early NIRS Monitoring to Predict Outcomes}

a. MR injury only: only 2 studies addressed.

Peng et al. [25] found consistently higher $\mathrm{CSO}_{2}$ values in moderately or severely encephalopathic infants receiving TH with later evidence of injury on MRI. In a cohort of 32 cooled infants with moderate encephalopathy, Goeral et al. [26] found no difference in $\mathrm{CSO}_{2}$ or FTOE values at $6 \mathrm{~h}$ in those with favourable or unfavourable MRI outcomes.

b. Long-term outcome only: only 1 study addressed.

Examining the ability of NIRS to predict long-term outcome, Ancora et al. [27] reported a trend towards a higher TOI at $6 \mathrm{~h}$ of life in infants with an adverse outcome at 18 months $(80.0 \% \pm 10.5$ vs. $66.9 \% \pm 7.0, p=$
$0.057)$, and this difference was statistically significant by 24 h of life $(79.7 \% \pm 9.4$ vs. $67.1 \% \pm 7.9, p=0.034)$. These infants all received $\mathrm{TH}$ for moderate or severe grades of encephalopathy.

c. Composite outcome of MR or long-term outcome: 4 studies addressed.

Both Lemmers et al. [22] and Niezen et al. [28]] found that early $\mathrm{CSO}_{2}$ and FTOE values did not differentiate between infants with a favourable or adverse outcome. They included infants with both moderate and severe grades of HIE undergoing TH. However, in both studies, the predictive ability of these NIRS measurements improved beyond the first $24-72 \mathrm{~h}$ of life at which time $\mathrm{cSO}_{2}$ was significantly higher in the adverse outcome group.

Nakamura et al. [29] also found no difference in $\mathrm{CSO}_{2}$ values at $6 \mathrm{~h}$ between those with a favourable and unfavourable outcome across all grades of encephalopathy. Mean CBV at $6 \mathrm{~h}$, however, was significantly higher in the infants who had an adverse outcome $(3.1 \pm 0.4$ vs. $2.4 \pm$ $0.5 \mathrm{~mL} / 100 \mathrm{~g}$ brain, $p=0.03$ ). At $24 \mathrm{~h}$ after birth, both $\mathrm{CSO}_{2}$ and $\mathrm{CBV}$ were higher in the infants with an adverse outcome, and they found that combining $\mathrm{CBV}$ and $\mathrm{CSO}_{2}$ at $24 \mathrm{~h}$ after birth had a $100 \%$ sensitivity, specificity, and positive and negative predictive values for neurological outcome.

Gucuyener et al. [30] noted that 3 infants in their cohort, all of which were cooled for moderate grade of encephalopathy, had low TOI and high FTOE before the initiation of TH. These same infants had repetitive seizures as confirmed by aEEG within the first few hours of life, suggesting increased oxygen consumption during seizures, and by the end of $\mathrm{TH}$, their values were within normal ranges. Although 2 of these infants had an abnormal MRI on day 7, all 3 had normal Bayleys scores at 2 years of age, highlighting the importance of long-term 
follow-up as an abnormal MRI is not synonymous with adverse neurodevelopmental outcome.

\section{Quality Assessment}

Quality of the included studies was assessed using the QUADAS-2 tool (Table 2). Regarding patient selection, it was unclear in some studies as to whether patients were consecutively enrolled or selected randomly, thus avoiding selection bias. In all studies, it was unclear as to whether the index test was interpreted without knowledge of the reference standard, and similar issues with blinding were present in some studies regarding the interpretation of the outcome data. Generally, concerns regarding applicability were low in the majority of studies.

\section{Discussion}

HIE is one of the top 10 contributors to the global burden of disease and has life-long effects for survivors and their families [31]. Use of $\mathrm{TH}$ in infants with moderate and severe encephalopathy has improved neurological outcomes. However, with significant disability still reported in over 1 million survivors [32], further improvement in therapy is required. Early NIRS monitoring may have an important role to play, not only in prediction of poor neurodevelopmental outcome but also in objective selection of infants for therapeutic intervention.

One of the main findings from this systematic review was the variation in methodology seen, specifically the device and sensors used, the measurements reported, and the outcome milestones assessed. Due to this heterogeneity, we were unable to perform a quantitative meta-analysis. In the 7 included studies, 6 different devices or sensors were used making it very difficult to draw firm conclusions. Although normative ranges for term infants have been suggested, these values have a narrow range and vary greatly between configurations of devices and sensors used $[33,34]$. Using the INVOS oximeter, for example, $\mathrm{CSO}_{2}$ values vary up to $10 \%$ depending on whether the neonatal or adult probes are used. In addition, the INVOS oximeter truncates values at $95 \%$, the range above which an adverse outcome may be expected and is an important point to consider when neonatal probes are used. Reapplication of a sensor in the same area may also result in variability of values of up to 6\% [35]. Furthermore, there is significant variation in the probe location used in the above studies, for example, frontal, parietal, and frontoparietal. Some studies obtained measurements bilaterally and some did not specify whether it was right or left.
This is important, as different NIRS systems have different penetration depths, so values may vary depending on the site used [36].

There was also variation in the short- and long-term outcome assessment methods used. Overall, 6 of the studies examined the ability of NIRS to predict brain injury on MRI, but the timing of these scans differed with some performing MRI in the first week of life and others into the second week of life. Long-term neurodevelopmental assessment also varied from 12 to 30 months of age. Furthermore, it is also unclear in many of the studies whether the authors were blinded to the NIRS values when assessing the outcome data and vice-versa, raising concerns for the potential for bias.

It is important, however, to also consider the timing of injury. As previously mentioned, timing of injury is frequently tacked to time of birth when in fact the timing of the sentinel event may vary and the resultant asphyxia may be acute, chronic, or acute-on-chronic [37], placing the infant at different stages along the injury pathway at time of birth. Nonetheless, NIRS provides an indirect measure of cerebral perfusion and oxygen consumption and has been shown to be very sensitive to acute changes in cerebral oxygenation [38]. Alterations in cerebral oxygenation and metabolism are pathognomonic of HIE, supporting the theoretical benefit of NIRS as a potential bedside biomarker. CBF has been shown to increase in the first $24 \mathrm{~h}$ following HI injury [19, 20]. In the pre-TH era, a low resistance index as measured by Doppler ultrasound was a good predictor of outcome in infants with HIE indicating impaired cerebral vasodilation and blood flow. This is no longer the case in the advent of $\mathrm{TH}$ [39]. However, in infants receiving TH, Wintermark et al. [21] found decreased CBF on day of life 1 in infants who later demonstrated hypoxic ischaemic injury on MRI. Therefore, early cerebral NIRS measurements of cerebral oxygenation, metabolism, and blood flow may contain important information that could aid in the prediction of outcome.

It is also necessary to address any potential confounding influencers on $\mathrm{CSO}_{2}$. $\mathrm{cSO}_{2}$ values comprised arterial, venous, and capillary saturations with a largely venous component (75\%). For this reason, care is needed in its interpretation as it portrays the fine balance between oxygen delivery and consumption and can thus be influenced by many variables including blood pressure, cardiac output, ventilator status, and concomitant medication. Nonetheless, trends in NIRS values can give important clues as to the haemodynamic status of the individual organs being monitored [40-42]. Sedation was 
described in 4 of the studies in which all infants received sedation as routine [27-30]. Three studies described the use of inotropes but found no difference in the use of inotropes between infants with a favourable or unfavourable outcome $[22,25,26]$. The impact of TH on NIRS is unclear. TH is thought to reduce metabolism and thus may decrease oxygen extraction. However, TH is also known to cause bradycardia which may influence cardiac output and thus oxygen delivery. Only Lemmers et al. [22] and Nakamura et al. [29] provided $\mathrm{cSO}_{2}$ values during both hypothermia and normothermia; however, they did not address the impact of TH on cerebral oxygenation.

Previous studies have shown that $\mathrm{cSO}_{2}$ levels beyond $24 \mathrm{~h}$ of life correlate with MRI findings and long-term neurodevelopmental outcome [22, 23]. Persistently higher $\mathrm{CSO}_{2}$ values are seen in infants with subsequent MRI injury and poor neurological outcomes [23, 43]. We set out to determine if early cerebral NIRS monitoring before $6 \mathrm{~h}$ of life could predict both short- and long-term outcomes and thereby aid in the identification of infants who would benefit most from TH. Although 2 studies demonstrated higher $\mathrm{CSO}_{2}$ and $\mathrm{CBF}$ values before $6 \mathrm{~h}$ in infants with adverse outcomes, in the majority, this difference was not significant until beyond $24 \mathrm{~h}$ of life [25-30].

Cerebral NIRS may be used in combination with blood pressure to assess autoregulation in infants with HIE [44]. Studies have used NIRS to determine the optimal mean arterial blood pressure at which autoregulation is maintained. Studies to date have found that infants who spent longer outside this optimal pressure range had a greater risk of adverse outcome [45]. This approach did not form part of our review, but information pertaining to early cerebral autoregulation could be beneficial in enabling early identification of infants at risk.

NIRS can also be used in combination with other modalities such as EEG or aEEG. As discussed, aEEG in the first $6 \mathrm{~h}$ of life was the most useful tool in predicting outcome in the pre-TH era. Although its predictive ability is now best seen at $48 \mathrm{~h}$ following the introduction of $\mathrm{TH}$ [12], the combination of NIRS and aEEG in the first $6 \mathrm{~h}$ of life may be useful in predicting outcome and thus the need for TH. Lemmers et al. [22] found that when aEEG and $\mathrm{CSO}_{2}$ scores were combined, both positive and negative predictive values improved to $70-91 \%$ and $90-100 \%$, respectively. Goeral et al. [26] found that a combination of both $\mathrm{cSO}_{2}$ and aEEG predicted MRI outcome as early as $18 \mathrm{~h}$.

Cerebral NIRS is both a feasible and acceptable monitoring tool in the neonatal unit. However, whether it re-

Does Early NIRS Monitoring in Neonates with HIE Predict Outcome? sults in a meaningful benefit to the neonatal HIE population remains to be seen. Based on limited available data, methodological heterogeneity in design, execution and available findings, early cerebral NIRS has not been evaluated for prediction of grades of HIE and is not associated with prediction of MRI evidence of brain injury or neurodevelopmental outcome.

The included studies in this review focused primarily on continuous wave NIRS to measure cerebral oxygenation. Other available methods include broadband NIRS or diffuse correlation spectroscopy which measure changes in cytochrome $\mathrm{c}$ oxidase and the cerebral metabolic rate of oxygen. These devices however are not commercially available and are therefore predominantly used in a research setting [46].

Whilst research to date has primarily focused on the value of NIRS monitoring as a trend monitor, signal processing techniques have shown that the NIRS signal itself may be rich in information [41]. Perhaps it is not the absolute values, rather the variation in values over time or quantitative analysis of the signal itself that is useful in outcome prediction. Quantitative analysis has the potential of providing an objective, continuous, and scalable analysis of the NIRS signal, but further research in this area is required. Future research should also focus on obtaining early measurements and including all grades of HIE. Combining NIRS with other monitoring modalities such as EEG and BP may enhance its predictive ability; however, it is also important that saturation values are measured using the same device, sensors, and methods.

This review is limited by the fact that it only includes articles written in English and was filtered to include human studies only as we wished to assess the usefulness of NIRS in clinical practice. As discussed, a small number of studies with varying methodologies were identified, so a meta-analysis was not feasible; however, this review was systematic and transparent. All full-text articles were independently reviewed, and we have clearly outlined reasons for inclusion and exclusion of studies in a PRISMA flowchart.

In conclusion, cerebral NIRS has the potential to be an important monitoring tool in infants with HIE. It can be used early after birth as it is quick and easy to apply and can provide continuous, non-invasive measurements. Unlike other monitoring modalities such as EEG, NIRS interpretation requires little training, and values are available instantaneously with a high sampling frequency. However, there are many questions to be answered before NIRS can be incorporated as an objective tool for identifying at-risk infants. Only 161 infants in total were in- 
cluded in the studies reviewed, and individually the studies had insufficient numbers to address their primary objectives. Only 4 infants with mild HIE were included in the studies, and therefore we are unable to determine whether there is a benefit to NIRS monitoring in this group. Furthermore, differing methodologies and conflicting results make it very difficult to draw any firm conclusions. There may be a trend towards higher $\mathrm{CSO}_{2}$ values at $6 \mathrm{~h}$ of life in infants with HIE who subsequently have adverse outcomes, but larger studies using a standardized approach and including all grades of encephalopathy are required. Furthermore, more precise measures of oxygen metabolism are likely required to identify the impairment in cerebral metabolism which occurs in the early stages of HIE prior to its incorporation into the neonatal unit as a predictive or decision-making tool.

\section{Acknowledgment}

The authors acknowledge the SFI Research Centre award and NCRC fellowship.

\section{Statement of Ethics}

The study is exempt from ethics committee approval as it involved a systematic review of the current published literature.

\section{Conflict of Interest Statement}

The authors have no conflicts of interest to declare.

\section{Funding Sources}

A.G. received a Clinical Research Fellowship grant from the National Children's Research Centre (D/18/6). A.P. received a research grant from the Health Research Board (CDA-2018-008). This research was also funded by an SFI Centre award (SFI/ $\mathrm{RC} / 2272)$.

\section{Author Contributions}

A.G. and A.P. conducted the literature search. All studies were screened by title and abstract by A.G. Remaining studies were screened for inclusion/exclusion criteria by A.G. and E.D. A.G. drafted the manuscript, and all authors contributed to reviewing and editing the manuscript.

\section{Data Availability Statement}

All data generated or analysed during this study are included in this article and its online supplementary material files. Further enquiries can be directed to the corresponding author.

\section{References}

1 Kurinczuk JJ, White-Koning M, Badawi N. Epidemiology of neonatal encephalopathy and hypoxic-ischaemic encephalopathy. Early Hum Dev. 2010;86(6):329-38.

2 Perlman JM. Pathogenesis of hypoxic-ischemic brain injury. J Perinatol. 2007;27(S1): S39-46.

3 Douglas-Escobar M, Weiss MD. Hypoxicischemic encephalopathy: a review for the clinician. JAMA Pediatr. 2015;169(4):397-403.

4 Sabir H, Scull-Brown E, Liu X, Thoresen M. Immediate hypothermia is not neuroprotective after severe hypoxia-ischemia and is deleterious when delayed by 12 hours in neonatal rats. Stroke. 2012;43(12):3364-70.

5 Thoresen M, Tooley J, Liu X, Jary S, Fleming $\mathrm{P}$, Luyt $\mathrm{K}$, et al. Time is brain: starting therapeutic hypothermia within three hours after birth improves motor outcome in asphyxiated newborns. Neonatology. 2013;104(3):22833.

6 Sarnat HB, Sarnat MS. Neonatal encephalopathy following fetal distress. A clinical and electroencephalographic study. Arch Neurol. 1976;33(10):696-705.
7 Conway JM, Walsh BH, Boylan GB, Murray DM. Mild hypoxic ischaemic encephalopathy and long term neurodevelopmental outcome: a systematic review. Early Hum Dev. 2018; 120:80-7.

8 Ruth VJ, Raivio KO. Perinatal brain damage: predictive value of metabolic acidosis and the Apgar score. BMJ. 1988;297(6640):24-7.

9 Natarajan G, Shankaran S, Laptook AR, Pappas A, Bann CM, McDonald SA, et al. Apgar scores at $10 \mathrm{~min}$ and outcomes at 6-7 years following hypoxic-ischaemic encephalopathy. Arch Dis Child Fetal Neonatal Ed. 2013; 98(6):F473-9.

10 Salvanos JB. Should we act on a high umbilical cord lactate in an otherwise healthy neonate? Arch Dis Child. 2020;105(2):200-2.

11 Thompson CM, Puterman AS, Linley LL, Hann FM, van der Elst CW, Molteno CD, et al. The value of a scoring system for hypoxic ischaemic encephalopathy in predicting neurodevelopmental outcome. Acta Paediatr. 1997;86(7):757-61.
12 Thoresen M, Hellström-Westas L, Liu X, de Vries LS. Effect of hypothermia on amplitude-integrated electroencephalogram in infants with asphyxia. Pediatrics. 2010;126(1): e131-9.

13 Rutherford M, Srinivasan L, Dyet L, Ward P, Allsop J, Counsell S, et al. Magnetic resonance imaging in perinatal brain injury: clinical presentation, lesions and outcome. Pediatr Radiol. 2006;36(7):582-92.

14 Robertson NJ, Wyatt JS. The magnetic resonance revolution in brain imaging: impact on neonatal intensive care. Arch Dis Child Fetal Neonatal Ed. 2004;89(3):F193-7.

15 Executive summary: neonatal encephalopathy and neurologic outcome, second edition. Report of the American College of Obstetricians and Gynecologists' Task Force on neonatal encephalopathy. Obstet Gynecol. 2014; 123(4):896-901.

16 Gano D, Chau V, Poskitt KJ, Hill A, Roland E, Brant R, et al. Evolution of pattern of injury and quantitative MRI on days 1 and 3 in term newborns with hypoxic-ischemic encephalopathy. Pediatr Res. 2013;74(1):82-7. 
17 McKinstry RC, Miller JH, Snyder AZ, Mathur A, Schefft GL, Almli CR, et al. A prospective, longitudinal diffusion tensor imaging study of brain injury in newborns. Neurology. 2002; 59(6):824-33.

18 Barkovich AJ, Miller SP, Bartha A, Newton N, Hamrick SE, Mukherjee P, et al. MR imaging, MR spectroscopy, and diffusion tensor imaging of sequential studies in neonates with encephalopathy. AJNR Am J Neuroradiol. 2006; 27(3):533-47.

19 Cope M, Delpy DT. System for long-term measurement of cerebral blood and tissue oxygenation on newborn infants by near infrared transillumination. Med Biol Eng Comput. 1988;26(3):289-94.

20 Meek JH, Elwell CE, McCormick DC, Edwards AD, Townsend JP, Stewart AL, et al. Abnormal cerebral haemodynamics in perinatally asphyxiated neonates related to outcome. Arch Dis Child Fetal Neonatal Ed. 1999;81(2):F110-5.

21 Wintermark P, Hansen A, Gregas MC, Soul J, Labrecque M, Robertson RL, et al. Brain perfusion in asphyxiated newborns treated with therapeutic hypothermia. AJNR Am J Neuroradiol. 2011;32(11):2023-9.

22 Lemmers PM, Zwanenburg RJ, Benders MJ, de Vries LS, Groenendaal F, van Bel F, et al. Cerebral oxygenation and brain activity after perinatal asphyxia: does hypothermia change their prognostic value? Pediatr Res. 2013; 74(2): $180-5$.

23 Jain SV, Pagano L, Gillam-Krakauer M, Slaughter JC, Pruthi S, Engelhardt B. Cerebral regional oxygen saturation trends in infants with hypoxic-ischemic encephalopathy. Early Hum Dev. 2017;113:55-61.

24 Liberati A, Altman DG, Tetzlaff J, Mulrow C, Gøtzsche PC, Ioannidis JP, et al. The PRISMA statement for reporting systematic reviews and meta-analyses of studies that evaluate health care interventions: explanation and elaboration. PLoS Med. 2009; 6(10): e1000100-34.

25 Peng S, Boudes E, Tan X, Saint-Martin C, Shevell M, Wintermark P. Does near-infrared spectroscopy identify asphyxiated newborns at risk of developing brain injury during hypothermia treatment? Am J Perinatol. 2015; 32(6):555-64.

26 Goeral K, Urlesberger B, Giordano V, Kaspri- an G, Wagner M, Schmidt L, et al. Prediction of outcome in neonates with HIE grade II: Role of aEEG and cerebral oxygen saturation measured by NIRS. Neonatology. 2017; 112(3):193-202.

27 Ancora G, Maranella E, Grandi S, Sbravati F, Coccolini E, Savini S, et al. Early predictors of short term neurodevelopmental outcome in asphyxiated cooled infants. A combined brain amplitude integrated electroencephalography and near infrared spectroscopy study. Brain Dev. 2013;35(1):26-31.

28 Niezen CK, Bos AF, Sival DA, Meiners LC, ter Horst HJ. Amplitude-integrated EEG and cerebral near-infrared spectroscopy in cooled, asphyxiated infants. Am J Perinatol. 2018; 35(9):904-10.

29 Nakamura S, Koyano K, Jinnai W, Hamano S, Yasuda S, Konishi Y, et al. Simultaneous measurement of cerebral hemoglobin oxygen saturation and blood volume in asphyxiated neonates by near-infrared time-resolved spectroscopy. Brain Dev. 2015;37(10):925-32.

30 Gucuyener K, Beken S, Ergenekon E, Soysal S, Hirfanoglu I, Turan O, et al. Use of amplitude-integrated electroencephalography (aEEG) and near infrared spectroscopy findings in neonates with asphyxia during selective head cooling. Brain Dev. 2012;34(4):2806

31 GBD 2013 Mortality and Causes of Death Collaborators. Global, regional, and national age-sex specific all-cause and cause-specific mortality for 240 causes of death, 1990-2013: a systematic analysis for the Global Burden of Disease Study 2013. Lancet. 2015;385(9963): $117-71$.

32 Lee AC, Kozuki N, Blencowe H, Vos T, Bahalim A, Darmstadt GL, et al. Intrapartumrelated neonatal encephalopathy incidence and impairment at regional and global levels for 2010 with trends from 1990. Pediatr Res. 2013;74 Suppl 1(Suppl 1):50-72.

33 Tina LG, Frigiola A, Abella R, Artale B, Puleo G, D’Angelo S, et al. Near infrared spectroscopy in healthy preterm and term newborns: correlation with gestational age and standard monitoring parameters. Curr Neurovasc Res. 2009;6(3):148-54.

34 Kleiser S, Nasseri N, Andresen B, Greisen G, Wolf M. Comparison of tissue oximeters on a liquid phantom with adjustable optical properties. Biomed Opt Express. 2016;7(8):297392.
35 Greisen G. Is near-infrared spectroscopy living up to its promises? Semin Fetal Neonatal Med. 2006;11(6):498-502.

36 Gunadi S, Leung TS, Elwell CE, Tachtsidis I. Spatial sensitivity and penetration depth of three cerebral oxygenation monitors. Biomed Opt Express. 2014;5(9):2896-912.

37 Westgate JA, Gunn AJ, Gunn TR. Antecedents of neonatal encephalopathy with fetal acidaemia at term. Br J Obstet Gynaecol. 1999;106(8):774-82.

38 Zhang D, Hou X, Liu Y, Zhou C, Luo Y, Ding $\mathrm{H}$. The utility of amplitude-integrated EEG and NIRS measurements as indices of hypoxic ischaemia in the newborn pig. Clin Neurophysiol. 2012;123(8):1668-75.

39 Elstad M, Whitelaw A, Thoresen M. Cerebral resistance index is less predictive in hypothermic encephalopathic newborns. Acta Paediatr. 2011;100(10):1344-9.

40 Wong FY, Barfield CP, Campbell L, Brodecky VA, Walker AM. Validation of cerebral venous oxygenation measured using near-infrared spectroscopy and partial jugular venous occlusion in the newborn lamb. J Cereb Blood Flow Metab. 2008;28(1):74-80.

41 O’Toole JM, Kenosi M, Finn D, Boylan GB, Dempsey EM. Features of cerebral oxygenation detects brain injury in premature infants. Annu Int Conf IEEE Eng Med Biol Soc. 2016;2016:3614-7.

42 Garvey AA, Kooi EMW, Smith A, Dempsey EM. Interpretation of cerebral oxygenation changes in the preterm infant. Children. 2018; 5(7):94.

43 Toet MC, Lemmers PM, van Schelven LJ, van Bel F. Cerebral oxygenation and electrical activity after birth asphyxia: their relation to outcome. Pediatrics. 2006;117(2):333-9.

44 Verhagen EA, Hummel LA, Bos AF, Kooi EM. Near-infrared spectroscopy to detect absence of cerebrovascular autoregulation in preterm infants. Clin Neurophysiol. 2014; 125(1):47-52.

45 Lee JK, Poretti A, Perin J, Huisman TAGM, Parkinson C, Chavez-Valdez R, et al. Optimizing cerebral autoregulation may decrease neonatal regional hypoxic-ischemic brain injury. Dev Neurosci. 2017;39(1-4):248-56.

46 Mitra S, Bale G, Meek J, Tachtsidis I, Robertson NJ. Cerebral near infrared spectroscopy monitoring in term infants with hypoxic ischemic encephalopathy: a systematic review. Front Neurol. 2020;11(393):393. 